\title{
Real-World Efficacy and Safety of Mycophenolate Mofetil in Active Moderate-to-Sight-Threatening Thyroid Eye Disease
}

\author{
Nicole Quah Qin Xian' \\ Ahmed Alnahrawy ${ }^{2}$ \\ Rashmi Akshikar ${ }^{2}$ \\ Vickie Lee ${ }^{2,3}$ \\ 'Department of Stroke Medicine, \\ Charing Cross Hospital, Imperial College \\ Healthcare NHS Trust, London, UK; \\ ${ }^{2}$ Department of Ophthalmology, \\ Western Eye Hospital, Imperial College \\ Healthcare NHS Trust, London, UK; \\ ${ }^{3}$ Department of Surgery \& Cancer, \\ General Surgery, Faculty of Medicine, \\ Imperial College London, London, UK
}

\begin{abstract}
Purpose: There is no universal consensus on second-line agents for the treatment of moderate/severe to sight-threatening thyroid eye disease (TED) to maintain remission after first-line intravenous methylprednisolone (IVMP). This study investigates the efficacy and safety of mycophenolate mofetil (MMF) in TED patients in a real-world setting and over a longer period than previous randomized controlled trials.
\end{abstract}

Methods: A retrospective cohort study of TED patients with active moderate/severe to sight-threatening TED seen over a 4-year period. Data collected were visual acuity (VA), Clinical Activity Score (CAS), Gorman Diplopia scores, MMF dosing and side effects at 24, 52 and 78 weeks. Clinical efficacy was defined as an absence of relapse: no decline in best corrected LogMAR VA, no need for further steroids, no increase in CAS of $\geq 2$.

Results: Out of 23 patients, 20 patients were included in this study. 10\% (2/20) stopped MMF before 24 weeks. Median duration of MMF treatment was 76 weeks (1-140 weeks). $55 \%(11 / 20)$ had dysthyroid optic neuropathy (DON). In those with active moderate-severe TED without DON, clinical efficacy was seen in $100 \%(8 / 8)$ at 24 weeks, $87.5 \%(7 / 8)$ at 52 weeks, and $83.3 \%(5 / 6)$ at 78 weeks, with CAS decreasing from a baseline of $2.78 \pm 1.99$ to $0.50 \pm 0.58$ at 24 weeks, $0.50 \pm 0.82$ at 52 weeks and $1.00 \pm 1.30$ at 78 weeks. In DON, improvements were seen in $90 \%(9 / 10)$ at 24 weeks, $100 \%(7 / 7)$ at 52 weeks and $100 \%$ $(4 / 4)$ at 78 weeks, with significantly reduced CAS scores from $2.55 \pm 1.54$ to $0.83 \pm 1.27,1.00$ \pm 1.17 and $0.63 \pm 0.95$ at 24,52 and 78 weeks, respectively. Gorman score, VA and soft tissue inflammation parameters also improved throughout. There were two significant side effects over the treatment period.

Conclusion: MMF appears to be an effective and safe second-line immunosuppressive agent. Further studies aimed at elucidating optimal dosing regimens and ideal treatment duration will prove helpful.

Keywords: Graves orbitopathy, dysthyroid optic neuropathy, CellCept

\section{Introduction}

Thyroid Eye Disease (TED) is a disfiguring and occasionally sight-threatening autoimmune orbital inflammation occurring predominantly in patients with Graves' hyperthyroidism. About two thirds of TED patients have clinically mild disease that requires supportive measures ${ }^{1,2}$ including achievement of euthyroidism, smoking cessation, selenium supplementation and tear supplements. ${ }^{2}$ Those with moderate-severe and sight-threatening disease usually benefit from timely immunosuppression of their eye disease to decrease the eventual disease burden and
Correspondence: Vickie Lee

Department of Ophthalmology, Western Eye Hospital, Imperial College Healthcare NHS Trust, I53-173 Marylebone Road, Marylebone, London, NWI 5QH, UK

Tel +44203312 6666

Email vickie.lee@nhs.net 
prevent visual loss. ${ }^{3}$ Regardless of disease severity, it is well known that TED inflicts significant psychological, occupational and financial burden. ${ }^{4}$

Medical management of TED continues to pose a challenge for clinicians. Most patients with moderatesevere and sight-threatening dysthyroid optic neuropathy (DON) disease in the UK are treated in line with the European Group of Graves' Orbitopathy (EUGOGO) recommendations with a 12-week course of weekly intravenous methylprednisolone (IVMP) as a first-line treatment for active moderate-severe TED, with higher initiation doses used for $\mathrm{DON}^{3}$ However, around one third of patients experience relapse after the first-line IVMP treatment. ${ }^{5}$ As TED is generally deemed a selflimiting condition that has an inflammatory duration of up to 12 to 18 months, there is a role for second-line consolidation treatment modalities including orbital radiotherapy or biological and non-biological immunosuppressive agents. ${ }^{3}$ There is no general consensus surrounding the optimal consolidation treatment. We describe our experience with mycophenolate mofetil (MMF) in our multidisciplinary thyroid eye (MDTED) clinics in an ethnically diverse patient cohort in London over a 4-year period.

\section{Materials and Methods}

Patients treated with MMF were identified through a retrospective audit of our MDTED clinics at Imperial College Healthcare NHS Trust. The MDTED was led by Consultant Ophthalmic and Oculoplastic Surgeon (VL) and Immunosuppression Specialist (RA) and included consultant ophthalmologists, endocrinologists and orthoptists (for measurement of ocular motility). Data was collected for all patients from the inception of the clinic in 2016 . Referrals for MMF treatment also came from two other associated MDTED clinics: Central Middlesex Hospital (CMH, 2011) and Charing Cross Hospital (CXH, 2017). The database used in this audit was directly populated and verified by a board round consensus of all the MDTED consultants working in the clinic. Additional clinical data were collected from the electronic record systems; this included clinic letters, biochemical and radiology results. Database lock was on 1st May 2020, before the initiation of the analysis. This retrospective audit was registered and approved by Imperial College Healthcare NHS Trust's Audit Department (ref: OPH_029); data were anonymized and confidentiality was maintained throughout. This study adhered to the tenets of the Declaration of Helsinki.
Our inclusion criteria comprised patients aged $>18$ years with moderate-severe TED who had received firstline IVMP treatment (using the EUGOGO protocol) over a 12-week period and started on MMF during or soon after IVMP treatment. Patients were excluded if they had inadequate health records, with no documentation available between our proposed timepoints of 24 weeks, 52 weeks, and 78 weeks after MMF initiation. As most of our patients started MMF after IVMP treatment, they had already been pre-screened for immunosuppression.

All patients had at least one endocrine assessment by an Endocrinologist, with optimization of thyroid status, where appropriate. Hyperthyroidism was treated with a titration or "block and replace" regimen, using carbimazole (CBZ) as first-line or propylthiouracil (PTU) as second-line; with thyroxine replacement added in the "block and replace" model. Hypothyroid patients and postthyroidectomy patients were treated with thyroxine replacement as guided by their thyroid function.

All patients were assessed for TED severity and activity by a Consultant Ophthalmologist through documentation of their symptoms, orbital and ocular examination and the Clinical Activity Score (CAS). ${ }^{6}$ Most of our patients also underwent magnetic resonance imaging (MRI) to assess the level of radiological inflammation. Those patients with TED of at least moderate severity, as determined by their required first-line IVMP, were considered to have clinically active TED. The severity of the disease was graded according to the EUGOGO consensus statement. $^{3}$

All of our TED patients who had received IVMP also received detailed counselling regarding whether to adopt a "treat and extend" approach after terminating IVMP where second-line treatment was only started if there were clinical signs of relapse, or to commence secondline treatment and/or orbital radiotherapy during their 12week course of IVMP. Usually, most of our DON patients would progress to either orbital radiotherapy and/or MMF, especially for those who required urgent orbital decompression during the early phase of their treatment. For those individuals with severe diplopia from restrictive myopathy or sight-threatening disease, we aimed to start MMF whilst they were still receiving their 12-week IVMP treatment.

MMF regimens in our MDTED clinics usually comprised an initiation phase (500 $\mathrm{mg}$ increasing up to $2 \mathrm{~g}$ ), followed by a maintenance phase (doses of $\geq 1.5 \mathrm{~g}$ ), then a weaning phase (1 $\mathrm{g}$ tapered down to $750 \mathrm{mg}$ then 
$500 \mathrm{mg}$ ). The intention was to treat for 52 weeks, and we generally aimed to initiate the treatment during the 12week IVMP course to allow MMF efficacy to coincide with the termination of IVMP treatment. During the initiation phase, they had weekly full blood counts (FBC), fortnightly urea and electrolytes (U\&Es) and liver function tests (LFTs) until dose stabilization, then the above tests monthly.

The following data were retrospectively collected for each patient: gender, ethnicity, age at MMF initiation, smoking status, thyroid disease diagnosis, thyroid treatments received prior to $\mathrm{MMF}$ initiation and after $\mathrm{MMF}$ discontinuation, duration of thyroid disease and ophthalmopathy, and previous treatments for thyroid disease and TED. Duration of thyroid disease was defined as the period between clinical or biochemical thyroid function derangement and database lockdown. The onset of TED was defined as the first onset of signs or symptoms or the first visit to an ophthalmology clinic where the diagnosis was made.

Ophthalmic parameters recorded were CAS score, soft tissue involvement including pain and swelling, Gorman Score for diplopia, best corrected visual acuity (BCVA) in the worst eye and Ishihara color vision. These parameters were recorded at the first presentation to the eye clinic just prior to MMF initiation, at 24 weeks, 52 weeks, 78 weeks, or at point of MMF cessation, if stopped prior to 24 weeks. Diplopia severity was evaluated using the Gorman scoring system: ${ }^{7}$ no diplopia $=1$, intermittent diplopia $=2$, inconstant or gaze-evoked diplopia $=3$, continuous diplopia in the primary and reading position $=4$. BCVA was converted to LogMAR score equivalents. ${ }^{8}$ For patients with visual acuity scores worse than 1.0 on LogMAR, we assigned a maximum value of 1.0 to the following acuities of Counting Fingers (CF), Hand Motion (HM), Light Perception (LP), and No Light Perception (NPL). For individuals with DON, color vision was recorded as the number of color vision plates correctly identified out of the total number of plates tested, with fractions then converted to decimals for comparison of color vision changes over time, to give a maximum score of 1 .

Baseline laboratory measurements before MMF initiation were recorded where available, of: Thyroid Stimulating Hormone (TSH), free tetraiodothyronine (fT4), TSH-receptor antibodies (TRAbs), thyroid peroxidase antibodies (TPOAbs), alanine aminotransferase (ALT), prothrombin time (PT), serum creatinine and estimated Glomerular Filtration Rate (eGFR). In cases where baseline measurements immediately prior to MMF administration were unavailable, the most recent available measurement before MMF initiation was used. Blood pressure and biochemical parameters were monitored at baseline and throughout treatment, including $\mathrm{FBC}$, renal profile and electrolytes, liver function tests, and glycated hemoglobin (HbA1c).

The primary outcomes were measured at dates closest to 24, 52 and 78 weeks after treatment initiation. Ophthalmic parameters at these timepoints were compared to those measured prior to MMF initiation. Clinical efficacy of MMF was defined as: an absence of relapse during the period of treatment, with relapse was defined as (i) worsening of BCVA denoted by a LogMAR score of $>0.2$; (ii) requirement of a further course of IVMP or oral steroids; (iii) increment in CAS score by $\geq 2$; or iv) decision to increase in MMF dose by $\geq 1 \mathrm{~g}$ following attempts at tapering.

Secondary outcomes included: side effects or clinically significant worsening of laboratory parameters during MMF treatment duration. A side effect is defined as any undesirable symptom or sign occurring after the start of MMF treatment, regardless of its relation to MMF. A significant side effect was defined as any medical occurrence relating to $\mathrm{MMF}$ treatment which required hospital admission or permanent MMF discontinuation. Significant derangement in any of the blood measurements over the course of MMF treatment was considered a side effect.

\section{Statistical Analysis}

Continuous variables were expressed as the mean \pm standard deviation (SD), with duration expressed as median (range). Categorical variables were expressed as frequency and percentage of total population. Welch's $t$-test was used to compare continuous variables for the population at the four defined timepoints. All statistical analyses were performed using the Data Analysis Toolkit on Microsoft Excel. Statistical significance is defined as $\mathrm{p}<0.05$.

\section{Results}

\section{Patient Demographics}

Twenty-three patients received MMF for TED treatment. 3 were excluded due to lack of documentation. $60 \%(12 / 20)$ of our cohort were female, and the mean age was 50.9 years (31-79 years, Table 1). 50\% (10/20) were documented to be of White ethnicity. $75 \%(15 / 20)$ of individuals were either ex-smokers $(70 \%, 14 / 20)$ or current smokers $(5 \%, 1 / 20)$. 
Table I Demographics and Baseline Characteristics of Our Cohort of 20 Individuals

\begin{tabular}{|c|c|c|}
\hline \multicolumn{2}{|l|}{ n } & 20 \\
\hline \multicolumn{2}{|l|}{ Sex (male: female) } & $8: 12$ \\
\hline \multicolumn{2}{|l|}{ Median age, years (range) } & $50.9(3 \mid-79)$ \\
\hline \multirow[t]{5}{*}{ Ethnicity } & White (Irish, British), \% & $10(50)$ \\
\hline & Black, African, Caribbean, Black British, \% & $3(15)$ \\
\hline & Asian or Asian British, \% & $3(15)$ \\
\hline & Mixed, \% & $2(10)$ \\
\hline & Other, \% & $2(10)$ \\
\hline \multirow[t]{3}{*}{ Smoking status } & Never smoked, \% & $5(25)$ \\
\hline & Ex-smoker, \% & $14(70)$ \\
\hline & Current smoker, \% & I (5) \\
\hline \multicolumn{2}{|l|}{ Median duration of Thyroid Disease, years (range) } & $2.9(0.8-61.7)$ \\
\hline \multicolumn{2}{|l|}{ Median duration of TED, years (range) } & $2.9(0.8-23.3)$ \\
\hline \multirow[t]{6}{*}{ Thyroid Disease } & Graves' hyperthyroidism, \% & $17(85)$ \\
\hline & Hashitoxicosis, \% & I (5) \\
\hline & Euthyroid, \% & I (5) \\
\hline & Thyroid resistance, \% & I (5) \\
\hline & Hashimoto’s, \% & $0(0)$ \\
\hline & Hypothyroidism, \% & $0(0)$ \\
\hline \multirow[t]{3}{*}{ Thyroid Treatment before MMF } & Anti-thyroid treatment ${ }^{\mathrm{a}}, \%$ & $13(65)$ \\
\hline & Thyroid hormone replacement, \% & $4(20)$ \\
\hline & No treatment, \% & $3(15)$ \\
\hline \multirow[t]{6}{*}{ Previous Treatments } & IVMP for TED, \% & $20(100)$ \\
\hline & Oral prednisolone for TED, \% & $4(20)$ \\
\hline & Orbital radiotherapy, \% & $7(35)$ \\
\hline & Optic nerve decompression, \% & II (55) \\
\hline & Radioactive iodine, \% & $3(15)$ \\
\hline & Thyroidectomy, \% & $2(10)$ \\
\hline \multicolumn{2}{|l|}{ Individuals with DON diagnosis, n (\%) } & II (55) \\
\hline \multirow[t]{8}{*}{ Biochemical and Immunological Characteristics before MMF } & $\mathrm{TSH}, \mathrm{mU} / \mathrm{L}(\mathrm{mean} \pm \mathrm{SD})$ & $5.5 \pm 15.5$ \\
\hline & $\mathrm{fT} 4, \mathrm{pmol} / \mathrm{L}(\operatorname{mean} \pm \mathrm{SD})$ & $15.3 \pm 10.4$ \\
\hline & TRAbs positive, \% & $12(60)$ \\
\hline & TPOAbs positive, \% & $0(0)$ \\
\hline & ALT, U/L (mean $\pm S D)$ & $29.1 \pm 13.5^{b}$ \\
\hline & PT, seconds (mean \pm SD) & $12.5 \pm 1.4$ \\
\hline & Serum creatinine, $\mu \mathrm{mol} / \mathrm{L}(\operatorname{mean} \pm \mathrm{SD})$ & $69.7 \pm 12.8$ \\
\hline & eGFR, $\mathrm{mL} / \mathrm{min} / 1.73 \mathrm{~m}^{2}(\operatorname{mean} \pm \mathrm{SD})$ & $84.8 \pm 10.9$ \\
\hline
\end{tabular}

Notes: ${ }^{a}$ Anti-thyroid treatments include: Titration (Carbimazole or Propylthiouracil) or Block and Replace (Carbimazole/propylthiouracil and levothyroxine). ${ }^{\mathrm{b}} \mathrm{I}$ sample excluded with ALT: 422 which had risen secondary to IVMP use. Decision was made to proceed with MMF initiation due to benefits outweighing risks.

Abbreviations: ALT, alanine aminotransferase; eGFR, estimated glomerular filtration rate; fT4, free tetraiodothyrodine; IVMP, iv methylprednisolone; MMF, mycophenolate mofetil; PT, prothrombin time; TED, thyroid eye disease; TPOAbs, thyroid peroxidase antibodies; TRAbs, TSH-receptor antibodies; TSH, thyroid-stimulating hormone.

The median duration of thyroid disease and TED was 2.9 years ( $0.8-61.7$ years) and 2.9 years $(0.8-23.3$ years) respectively. $85 \%(17 / 20)$ had Graves' disease with $60 \%$ $(12 / 20)$ TSH-receptor antibody positive. $65 \%(13 / 20)$ of individuals were on anti-thyroid treatment in a titration or block and replace regimen, whilst $20 \%(4 / 20)$ were on thyroid hormone replacement therapy, and 15\% (3/20) did not require ongoing treatment. All individuals received IVMP as a first-line immunosuppression, with 20\% (4/20) also receiving oral steroids. $35 \%(7 / 20)$ had undergone 


\section{Duration of Mycophenolate Mofetil Treatment for 20 Patients}

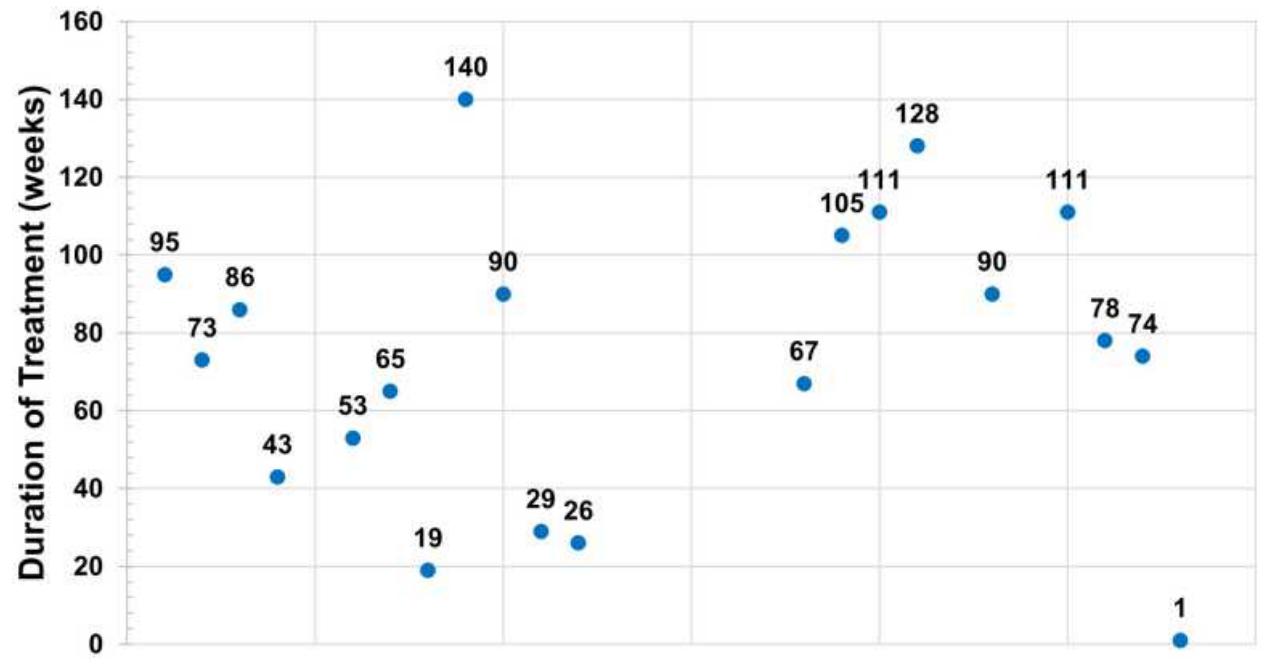

Figure I Duration of mycophenolate mofetil treatment for 20 patients.

previous orbital radiotherapy, whilst $55 \%$ of individuals $(11 / 20)$ had undergone previous orbital decompression. $15 \%(3 / 20)$ had previously received radioactive iodine (RAI) treatment and 10\% (2/20) had undergone thyroidectomy. Prior to MMF initiation, 65\% (13/20) of our patients were euthyroid, with $25 \%(5 / 20)$ documented to be hyperthyroid, and $10 \%(2 / 20)$ hypothyroid at the point of their first MDT visit. There were no biochemical results indicating renal dysfunction. One individual had slightly deranged liver function tests secondary to IVMP use.

\section{Patient Responses to MMF}

The median duration of MMF treatment at the point of database lockdown was 76 weeks (1-140 weeks) (Figure 1). 90\% (18/20) had received MMF for at least 24 weeks; 75\% (15/20) for at least 52 weeks and 50\% (10/20) for at least 78 weeks. Two individuals received MMF for less than 24 weeks (19 weeks, 1 week). At 24 weeks, average MMF dose was $1722 \mathrm{mg}$, with 22.2\% (4/18) of individuals receiving $1500 \mathrm{mg}$ and $66.7 \%(12 / 18)$ receiving $2000 \mathrm{mg}$ or more. At 52 weeks, mean dose was $1339 \mathrm{mg}$; $13 \%(2 / 15)$ were on $1500 \mathrm{mg}$ and $40 \%(6 / 15)$ on $2000 \mathrm{mg}$. At 78 weeks, mean dose was $1472 \mathrm{mg}, 30 \%$ (3/10) were on $1500 \mathrm{mg}$ and $30 \%(3 / 10)$ were receiving $2000 \mathrm{mg}$ or more.

At baseline, there were 9/20 (45\%) individuals with active moderate-severe TED without sight-threatening DON, and 11/20 (55\%) with a DON diagnosis. By 24 weeks, individuals with DON comprised 10/18 (55.6\%) of the cohort, whereas this dropped to $7 / 15(46.7 \%)$ by 52 weeks and $4 / 10(40 \%)$ by 78 weeks. The changing demographics of treatments received by each cohort at each timepoint are depicted in Figure 2.

\section{Baseline Findings}

In the 9 individuals with active moderate-severe TED without DON, the mean CAS prior to MMF treatment was $2.78 \pm 1.99$. At baseline, the most common ophthalmic feature was soft tissue involvement $(88.9 \%, 8 / 9)$, characterized by pain $(77.8,7 / 9)$. The mean Gorman score was $2.33 \pm 1.32$. The mean BCVA score on the LogMAR chart for the worst-affected eye was $0.13 \pm 0.19$ (Table 2).

In the 11 individuals with DON, mean baseline CAS was $2.55 \pm 1.54$. Soft tissue involvement was the most common ophthalmic feature $(90 \%, 9 / 10)$, with $88.9 \%(8 /$ 9) individuals reporting pain. Mean Gorman score was 2.64 \pm 0.99 ; mean BCVA was $0.45 \pm 0.42$ and mean color vision score for those with DON was $0.79 \pm 0.37$ (Table 2).

\section{Weeks (I8 Patients)}

In those without DON (8 individuals; 7 ex-smokers, 1 nonsmoker), the mean CAS was $0.50 \pm 0.58$. This was a statistically significant improvement from the baseline CAS of $2.78 \pm 1.99(p=0.01)$. There was a general trend in improvement in all other ophthalmic parameters. Overall, $100 \%(8 / 8)$ of individuals exhibited improvements when assessed after 24 weeks of MMF treatment, with no relapses (Table 3 ).

In DON cases (10 individuals, 7 ex-smokers, 3 nonsmokers), the mean CAS was $0.83 \pm 1.27$, a significant decrease from $2.55 \pm 1.54$ baseline $(\mathrm{p}=0.004)$. Other ophthalmic parameters were also improved, with 90\% (9/ 


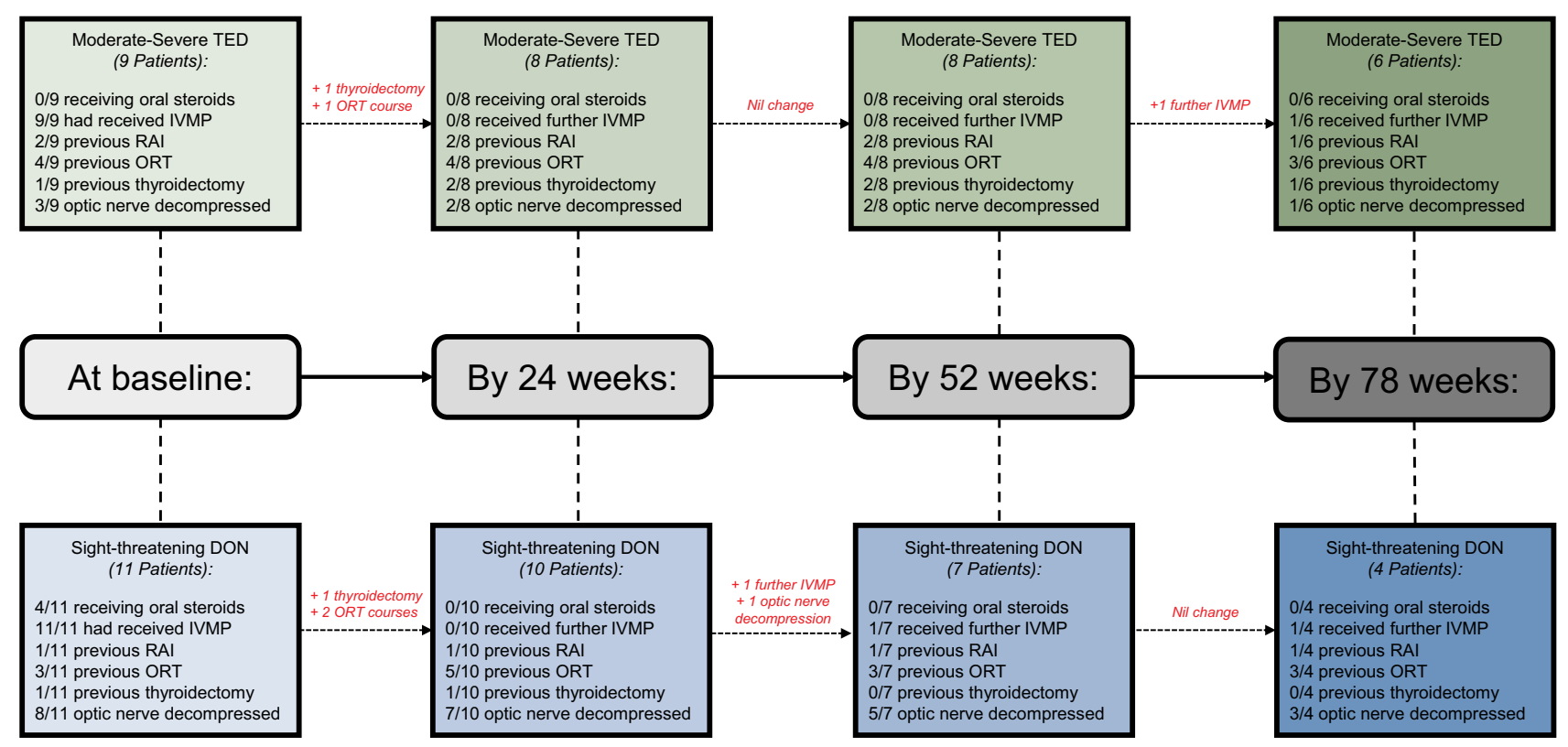

Figure 2 Flowchart demonstrating the treatments received by individuals with moderate-severe TED and those with DON at baseline, 24 weeks, 52 weeks and 78 weeks.

10) of individuals reporting improvements. One patient (10.0\%) had worsened BCVA with an increase of 0.3 LogMAR BCVA (Table 4).

\section{Weeks (I5 Patients)}

At 52 weeks, those without DON (8 individuals, 7 exsmokers, 1 non-smoker) had a mean CAS of $0.50 \pm 0.82$, a statistical improvement from baseline CAS ( $\mathrm{p}=0.016)$. There was maintenance of the general trend in improvement of ophthalmic parameters compared to baseline and overall, $87.5 \%$ (7/8) individuals improved after 52 weeks of MMF. 1 individual (12.5\%) experienced a relapse due to a decrement in BCVA $>0.2$ on LogMAR chart.

For individuals with DON (7 individuals, $4 \mathrm{ex}-$ smokers, 3 non-smokers), the mean CAS was $1.00 \pm 1.17$ which was significantly decreased from baseline $(\mathrm{p}=0.016)$. All 7 patients $(100 \%)$ reported improvements amongst their other ophthalmic parameters, although one (14.3\%) individual experienced a relapse by our definition during this same timeframe, due to provision of a further IVMP course.

\section{Weeks (I0 Patients)}

At 78 weeks, there were 6 individuals with moderatesevere TED without DON (5 ex-smokers, 1 smoker). Mean CAS was $1.00 \pm 1.30$ compared to the baseline of $2.78 \pm 1.99(\mathrm{p}=0.101)$. Overall, a response occurred in $83.3 \%(5 / 6)$ of those individuals still receiving MMF at 78 weeks. This was the stage when our MDTED clinics tried to wean patients off MMF in line with our regimen, and 1 individual (16.7\%) required a slower weaning off period with some increases in doses needed after a slight relapse in soft tissue symptoms.

For the remaining 4 individuals with DON (1 exsmoker, 3 non-smokers), mean CAS was $0.63 \pm 0.95$ which was significantly lower than baseline $(\mathrm{p}=0.006)$. All individuals experienced improvements in their ophthalmic parameters, though 1 individual (25\%) required a re-increase in MMF dose during the weaning process.

\section{Side Effects}

We recorded side effects in $65 \%(13 / 20)$ of our cohort, but most of these were mild. $10 \%(2 / 20)$ met the criteria for a significant side effect related to MMF treatment (Table 5) - one being viral pneumonia requiring hospital admission between the 24th and 52nd week and the other being MMF discontinuation due to development of severe gastrointestinal symptoms in the first 4 weeks in an individual who subsequently underwent bilateral orbital decompression, as well as orbital radiotherapy for their DON with eventual good outcome.

\section{Discussion}

As far as we are aware, there are few studies in the peerreviewed literature describing the use of MMF in a realworld clinical setting for TED treatment. In a recent UK 
Table 2 Ophthalmic Assessments for Individuals with Moderate-Severe TED and Sight-Threatening DON at Baseline, 24 Weeks, 52 Weeks, 78 Weeks

\begin{tabular}{|c|c|c|c|c|}
\hline & Before MMF & 24 Weeks & 52 Weeks & 78 Weeks \\
\hline \multicolumn{5}{|c|}{ Individuals with Active Moderate-Severe TED without Sight-Threatening DON } \\
\hline $\mathbf{n}$ & 9 & 8 & 8 & 6 \\
\hline CAS (mean \pm SD) & $2.78 \pm 1.99^{\mathrm{a}}$ & $0.50 \pm 0.58^{d}$ & $0.50 \pm 0.82^{g}$ & $1.00 \pm 1.30^{\mathrm{j}}$ \\
\hline Soft tissue involvement, \% & $8 / 9(88.9)$ & $2 / 5(40.0)$ & $4 / 7(57.1)$ & $3 / 5(60.0)$ \\
\hline Pain, \% & $7 / 9(77.8)$ & $\mathrm{I} / 5(20.0)$ & $2 / 5(40.0)$ & $2 / 5(40.0)$ \\
\hline Gorman Score (mean $\pm \mathrm{SD})$ & $2.33 \pm 1.32^{b}$ & $0.63 \pm 0.50^{\mathrm{e}}$ & $1.13 \pm 0.84^{\mathrm{h}}$ & $1.83 \pm 1.30^{k}$ \\
\hline$V A$ in worst eye (mean $\pm S D)$ & $0.13 \pm 0.19^{c}$ & $0.04 \pm 0.22^{f}$ & $0.11 \pm 0.27^{i}$ & $0.00 \pm 0.14^{\prime}$ \\
\hline \multicolumn{5}{|c|}{ Individuals with Sight-Threatening DON } \\
\hline $\mathbf{n}$ & II & 10 & 7 & 4 \\
\hline CAS (mean $\pm S D)$ & $2.55 \pm 1.54^{\mathrm{m}}$ & $0.83 \pm 1.27^{9}$ & $1.00 \pm 1.17^{\mathrm{u}}$ & $0.63 \pm 0.95^{y}$ \\
\hline Soft tissue involvement, \% & $9 / 10(90.0)$ & $3 / 10(30.0)$ & I/5 (20.0) & $2 / 4(50.0)$ \\
\hline Pain, \% & $8 / 9(88.9)$ & $3 / 10(30.0)$ & $3 / 7(42.9)$ & $0 / 4(0.0)$ \\
\hline Gorman Score (mean $\pm S D)$ & $2.64 \pm 0.99^{n}$ & $1.50 \pm 1.22^{r}$ & $1.43 \pm 1.29^{v}$ & $1.75 \pm 1.53^{z}$ \\
\hline$V A$ in worst eye (mean $\pm S D)$ & $0.45 \pm 0.42^{\circ}$ & $0.42 \pm 0.42^{s}$ & $0.40 \pm 0.45^{w}$ & $0.33 \pm 0.48^{\mathrm{aa}}$ \\
\hline Color vision (mean $\pm \mathrm{SD})$ & $0.79 \pm 0.37^{P}$ & $0.94 \pm 0.16^{\mathrm{t}}$ & $0.68 \pm 0.26^{x}$ & $0.98 \pm 0.03^{\mathrm{ab}}$ \\
\hline
\end{tabular}

Notes: a Mean Clinical Activity Score at baseline for $9 / 9$ individuals. ${ }^{b}$ Mean Gorman score at baseline for $9 / 9$ individuals. ${ }^{\mathrm{C}}$ Mean visual acuity at baseline for $9 / 9$ individuals. ${ }^{\mathrm{d}}$ Mean Clinical Activity Score at 24 weeks for $4 / 8$ individuals. ${ }^{e}$ Mean Gorman score at 24 weeks for $4 / 8$ individuals. ${ }^{\mathrm{f}}$ Mean visual acuity at 24 weeks for $8 / 8$ individuals. ${ }^{\mathrm{g}}$ Mean Clinical Activity Score at 52 weeks for $6 / 8$ individuals. ${ }^{\mathrm{h}}$ Mean Gorman score at 52 weeks for $6 / 8$ individuals. 'Mean visual acuity at 52 weeks for 8/8 individuals. 'Mean Clinical Activity Score at 78 weeks for $5 / 6$ individuals. ${ }^{k}$ Mean Gorman score at 78 weeks for $5 / 6$ individuals. 'Mean visual acuity at 78 weeks for $5 / 6$ individuals. ${ }^{m}$ Mean Clinical Activity Score at baseline for $9 / 1$ I individuals. ${ }^{n}$ Mean Gorman score at baseline for $10 / 1 \mathrm{I}$ individuals. ${ }^{\circ}$ Mean visual acuity at baseline for $\mathrm{I}$ I/I I individuals. ${ }^{~}$ Color vision score at baseline for I I/II individuals. ${ }^{9} \mathrm{Mean}$ Clinical Activity Score at 24 weeks for $9 / 10$ individuals. ' $M e a n$ Gorman score at 24 weeks for $5 / 10$ individuals. ${ }^{5}$ Mean visual acuity at 24 weeks for $10 / 10$ individuals. ${ }^{\mathrm{t}} \mathrm{Color}$ vision score at 24 weeks for $7 / 10$

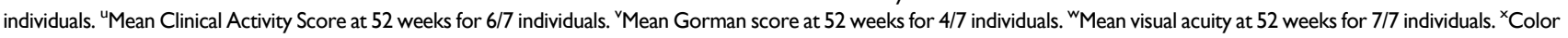
vision score at 52 weeks for $5 / 7$ individuals. ${ }^{y}$ Mean Clinical Activity Score at 78 weeks for $4 / 4$ individuals. ${ }^{2}$ Mean Gorman score at 78 weeks for $3 / 4$ individuals. ${ }^{\text {aa } M e a n ~ v i s u a l ~ a c u i t y ~ a t ~} 78$ weeks for $4 / 4$ individuals. ${ }^{\text {ab }}$ Color vision score at 78 weeks for $4 / 4$ individuals.

Abbreviations: CAS, Clinical Activity Score; DON, dysthyroid optic neuropathy; MMF, mycophenolate mofetil; SD, standard deviation; TED, thyroid eye disease; VA, visual acuity.

survey of TED services, it was found that there was significant geographical variation in the accessibility of second-line immunosuppression. ${ }^{9}$ Despite the advent of the recently targeted TED treatment Teprotumumab, ${ }^{2,10,11}$ due to its limited licensing and cost, there remains a distinct need for an effective and safe yet affordable second-line immunosuppression agent. Although previous randomized controlled prospective studies have investigated the role of mycophenolate in active moderate-severe TED, ${ }^{12,13}$ these studies specifically excluded individuals with sight-threatening DON, ${ }^{12,13}$ recent second-line immunosuppression, ${ }^{12,13}$ and previous orbital decompression. ${ }^{12}$ In normal clinical practice, a combination of these modalities is used to control severe and sight-threatening disease, and our population demographics reflect this. Our study examined the effects of MMF treatment in an ethnically diverse cohort for durations of up to 18 months, whereas previous studies had a final follow-up at 9 months in cohorts with less severe disease than ours. Our MMF cohort had a much higher proportion of smokers and ex-smokers (75\%) compared to all of the patients attending our MDTED clinics (43\%). ${ }^{14}$
We found it difficult to wean half of our patients off MMF even at 52 weeks as patients would complain of increased soft tissue swelling or pain upon dose reduction.

We found that the optimal duration of MMF treatment may lie between 24 and 78 weeks; this is similar to Ye et al's highly promising findings of a $91 \%$ response rate to MMF at 24 weeks, ${ }^{12}$ and higher than Kahaly et al's figure of $71 \%$ response to mycophenolate sodium at 24 weeks. ${ }^{13}$ Mycophenolate sodium (Myfortic) is an enteric-coated formulation of mycophenolate acid, whereas MMF (Cellcept) is a pro-drug of mycophenolate acid with higher oral bioavailability, which may explain the increased levels of clinical response observed with MMF over mycophenolate sodium. ${ }^{15}$ Of note, despite the current global pandemic of COVID-19 which has called for reviews over the necessity of existing immunosuppressive therapy, none of our patients terminated their MMF treatments.

Current literature reports that the most common side effects of MMF include gastrointestinal ${ }^{16}$ or hematological disorders, and infections. ${ }^{13,17}$ Sore throat was the most common side effect and we had 2 cases of mild gastrointestinal disturbance or hematological derangements 
Table 3 Ophthalmic Responses for Individuals with Moderate-Severe TED and Individuals with Sight-Threatening DON at 24 Weeks, 52 Weeks, 78 Weeks vs Baseline

\begin{tabular}{|c|c|c|c|}
\hline & 24 Weeks & 52 Weeks & 78 Weeks \\
\hline \multicolumn{4}{|c|}{ Individuals with Active Moderate-Severe TED without Sight-Threatening DON } \\
\hline Total $n$ & 8 & 8 & 6 \\
\hline CAS score available $n, \%$ & $4 / 8(50.0)$ & $6 / 8(75.0)$ & $5 / 6(83.3)$ \\
\hline Improved, \% & $3 / 8(37.5)$ & $5 / 8(62.5)$ & $4 / 6(66.7)$ \\
\hline Stable, \% & I/8 (12.5) & I/8 (I2.5) & $1 / 6(16.7)$ \\
\hline Deteriorated, \% & $0 / 8(0.0)$ & $0 / 8(0.0)$ & $0 / 6(0.0)$ \\
\hline Unknown, \% & $4 / 8(50.0)$ & $2 / 8(25.0)$ & $1 / 6(16.7)$ \\
\hline Soft tissue involvement $\mathrm{n}, \%$ & $2 / 5(40.0)$ & $4 / 7(57.1)$ & $3 / 5(60.0)$ \\
\hline Improved, \% & $3 / 8(37.5)$ & $3 / 8(37.5)$ & $2 / 6(33.3)$ \\
\hline Stable, \% & $2 / 8(25.0)$ & $3 / 8(37.5)$ & $2 / 6(33.3)$ \\
\hline Deteriorated, \% & $0 / 8(0.0)$ & I/8 (I2.5) & $1 / 6(16.7)$ \\
\hline Unknown, \% & $3 / 8(37.5)$ & I/8 (I2.5) & $1 / 6(16.7)$ \\
\hline Diplopia n, \% & $\mathrm{I} / 4(25.0)$ & $2 / 6(33.3)$ & $3 / 5(60.0)$ \\
\hline Improved, \% & $3 / 8(37.5)$ & $3 / 8(37.5)$ & $1 / 6(16.7)$ \\
\hline Stable, \% & $2 / 8(25.0)$ & $2 / 8(25.0)$ & $2 / 6(33.3)$ \\
\hline Deteriorated, \% & I/8 (I2.5) & I/8 (I2.5) & $2 / 6(33.3)$ \\
\hline Unknown, \% & $2 / 8(25.0)$ & $2 / 8(25.0)$ & $1 / 6(16.7)$ \\
\hline Pain n, \% & $\mathrm{I} / 5(20.0)$ & $2 / 5(40.0)$ & $2 / 5(40.0)$ \\
\hline Improved, \% & $3 / 8(37.5)$ & $3 / 8(37.5)$ & $2 / 6(33.3)$ \\
\hline Stable, \% & $2 / 8(25.0)$ & $2 / 8(25.0)$ & $3 / 6(50.0)$ \\
\hline Deteriorated, \% & $0 / 8(0.0)$ & $0 / 8(0.0)$ & $0 / 10(0.0)$ \\
\hline Unknown, \% & $3 / 8(37.5)$ & $3 / 8(37.5)$ & $1 / 6(16.7)$ \\
\hline Visual acuity in most affected eye $n, \%$ & $8 / 8(100.0)$ & $8 / 8(100.0)$ & $5 / 6(83.3)$ \\
\hline Improved, \% & $5 / 8(62.5)$ & $5 / 8(62.5)$ & $2 / 6(33.3)$ \\
\hline Stable, \% & $2 / 8(25.0)$ & $2 / 8(25.0)$ & $3 / 6(50.0)$ \\
\hline Deteriorated, \% & I/8 (12.5) & I/8 (I2.5) & $0 / 6(0.0)$ \\
\hline Unknown, \% & $0 / 8(0.0)$ & $0 / 8(0.0)$ & $1 / 6(16.7)$ \\
\hline Improvement in any Parameter $\mathrm{n}, \%$ & $8 / 8(100.0)$ & $7 / 8(87.5)$ & $5 / 6(83.3)$ \\
\hline \multicolumn{4}{|l|}{ Individuals with Sight-Threatening DON } \\
\hline Total $n$ & 10 & 7 & 4 \\
\hline CAS score available $n, \%$ & $9 / 10(90.0)$ & $6 / 7(85.7)$ & $4 / 4(100.0)$ \\
\hline Improved, \% & $7 / 10(70.0)$ & $5 / 7(7 I .4)$ & $3 / 4(75.0)$ \\
\hline Stable, \% & $1 / 10(10.0)$ & I/7 (14.3) & $\mathrm{I} / 4(25.0)$ \\
\hline Deteriorated, \% & $0 / 10(0.0)$ & $0 / 7(0.0)$ & $0 / 4(0.0)$ \\
\hline Unknown, \% & $2 / 10(20.0)$ & I/7 (I4.3) & $0 / 4(0.0)$ \\
\hline Soft tissue involvement $\mathrm{n}, \%$ & $3 / 10(30.0)$ & $\mathrm{I} / 5(20.0)$ & $2 / 4(50.0)$ \\
\hline Improved, \% & $6 / 10(60.0)$ & $3 / 7(42.9)$ & $3 / 4(75.0)$ \\
\hline Stable, \% & $4 / 10(40.0)$ & $2 / 7(28.6)$ & $0 / 4(0.0)$ \\
\hline Deteriorated, \% & $0 / 10(0.0)$ & $0 / 7(0.0)$ & I/4 (25.0) \\
\hline Unknown, \% & $0 / 10(0.0)$ & $2 / 7(28.6)$ & $0 / 4(0.0)$ \\
\hline Diplopia n, \% & $4 / 5(80.0)$ & $3 / 4(75.0)$ & $2 / 3(66.7)$ \\
\hline Improved, \% & $3 / 10(30.0)$ & $3 / 7(42.9)$ & I/4 (25.0) \\
\hline Stable, \% & $3 / 10(30.0)$ & I/7 (I4.3) & I/4 (25.0) \\
\hline Deteriorated, \% & $1 / 10(10.0)$ & I/7 (14.3) & $\mathrm{I} / 4(25.0)$ \\
\hline
\end{tabular}

(Continued) 
Table 3 (Continued).

\begin{tabular}{|c|c|c|c|}
\hline & 24 Weeks & 52 Weeks & 78 Weeks \\
\hline Unknown, \% & $3 / 10(30.0)$ & $2 / 7(28.6)$ & I/4 (25.0) \\
\hline Pain n, \% & $3 / 10(30.0)$ & $3 / 7(42.9)$ & $0 / 4(0.0)$ \\
\hline Improved, \% & $6 / 10(60.0)$ & $6 / 7(85.7)$ & $4 / 4(100.0)$ \\
\hline Stable, \% & $3 / 10(30.0)$ & $0 / 7(0.0)$ & $0 / 4(0.0)$ \\
\hline Deteriorated, \% & $0 / 10(0.0)$ & $0 / 7(0.0)$ & $0 / 4(0.0)$ \\
\hline Unknown, \% & $1 / 10(10.0)$ & I/7 (14.3) & $0 / 4(0.0)$ \\
\hline Visual acuity in most affected eye $n, \%$ & $10 / 10(100.0)$ & $7 / 7(100.0)$ & $4 / 4(100.0)$ \\
\hline Improved, \% & $5 / 10(50.0)$ & $3 / 7(42.9)$ & $\mathrm{I} / 4(25.0)$ \\
\hline Stable, $\%$ & $3 / 10(30.0)$ & $3 / 7(42.9)$ & I/4 (25.0) \\
\hline Deteriorated, \% & $2 / 10(20.0)$ & I/7 (I4.3) & I/4 (25.0) \\
\hline Unknown, \% & $0 / 10(0.0)$ & $0 / 7(0.0)$ & I/4 (25.0) \\
\hline Color Vision score available $n, \%$ & $7 / 10(70.0)$ & $5 / 7(71.4)$ & $4 / 4(100.0)$ \\
\hline Improved, \% & $2 / 10(20.0)$ & $2 / 7(28.6)$ & $0 / 4(0.0)$ \\
\hline Stable, $\%$ & $4 / 10(40.0)$ & $2 / 7(28.6)$ & $3 / 4(75.0)$ \\
\hline Deteriorated, \% & $0 / 10(0.0)$ & I/7 (14.3) & $\mathrm{I} / 4(25.0)$ \\
\hline Unknown, \% & $4 / 10(40.0)$ & $2 / 7(28.6)$ & $0 / 4(0.0)$ \\
\hline Improvement in any Parameter $\mathrm{n}, \%$ & $9 / 10(90.0)$ & $7 / 7(100.0)$ & $4 / 4(100.0)$ \\
\hline
\end{tabular}

Abbreviations: CAS, Clinical Activity Score; DON, dysthyroid optic neuropathy; TED, thyroid eye disease.

Table 4 Relapses in Those with Moderate-Severe TED and Sight-Threatening DON Occurring by 24 Weeks, 52 Weeks, 78 Weeks

\begin{tabular}{|c|c|c|c|}
\hline & 24 Weeks & 52 Weeks & 78 Weeks \\
\hline \multicolumn{4}{|c|}{ Individuals with Active Moderate-Severe TED without Sight-Threatening DON } \\
\hline Worse BCVA & 0 & 1 & 0 \\
\hline Further IVMP Doses & 0 & 0 & 0 \\
\hline Increase in CAS & 0 & 0 & 0 \\
\hline Up-titration of MMF & 0 & 0 & I \\
\hline Total Relapse, n (\%) & $0 / 8(0.0)$ & $1 / 8(12.5)$ & $1 / 6(16.7)$ \\
\hline \multicolumn{4}{|c|}{ Individuals with Sight-Threatening DON } \\
\hline Worse BCVA & I & 0 & 0 \\
\hline Further IVMP Doses & 0 & I & 0 \\
\hline Increase in CAS & 0 & 0 & 0 \\
\hline Up-titration of MMF & 0 & 0 & 1 \\
\hline Total Relapse, n (\%) & $1 / 10(10.0 \%)$ & I/7 (I4.3) & I/4 (25.0) \\
\hline
\end{tabular}

Abbreviations: BCVA, best corrected visual acuity; CAS, Clinical Activity Score; DON, dysthyroid optic neuropathy; IVMP, intravenous methylprednisolone; MMF, mycophenolate mofetil; TED, thyroid eye disease.

within our cohort. Significant side effects occurred in 2 patients (viral pneumonia and GI upset), the latter of which resulted in permanent cessation. The viral pneumonia required hospital admission and although MMF was temporarily suspended, it was not permanently discontinued. Both these patients had several other comorbidities; thus, these events may not necessarily have been directly MMF-related. Our other MMF permanent cessation occurring before 24 weeks was due to a switch to ciclosporin at 19 weeks. Overall, these findings suggest that the safety profile of MMF is comparable to the safety profile of oral and intravenous steroids ${ }^{18,19}$ and offers promising initial 
Table 5 Side Effects During Mycophenolate Mofetil Treatment for Our Cohort

\begin{tabular}{|c|c|c|c|c|c|c|c|c|c|}
\hline & \multicolumn{9}{|c|}{ Week } \\
\hline & 4 & 8 & 12 & 16 & 20 & 24 & 52 & 78 & $\begin{array}{c}\text { Post } \\
78\end{array}$ \\
\hline Infection, total & 2 & 1 & 0 & 1 & 0 & 0 & 7 & 1 & 0 \\
\hline Tonsillitis $^{\mathrm{a}}$ & 2 & 1 & 0 & 0 & 0 & 0 & 4 & 0 & 0 \\
\hline Chest infection ${ }^{\mathrm{b}}$ & 0 & 0 & 0 & I & 0 & 0 & 2 & 0 & 0 \\
\hline Viral labyrinthitis & 0 & 0 & 0 & 0 & 0 & 0 & 1 & 0 & 0 \\
\hline Urinary tract infection & 0 & 0 & 0 & 0 & 0 & 0 & 0 & 1 & 0 \\
\hline \multicolumn{10}{|l|}{ Gastrointestinal and } \\
\hline Hepatobiliary, total & 3 & 0 & 0 & 0 & 1 & 0 & 2 & 1 & 0 \\
\hline Nausea & 0 & 0 & 0 & 0 & 0 & 0 & 2 & 0 & 0 \\
\hline Mouth ulceration & 1 & 0 & 0 & 0 & 0 & 0 & 0 & 0 & 0 \\
\hline Change in bowel habit ${ }^{c}$ & 0 & 0 & 0 & 0 & 1 & 0 & 0 & 1 & 0 \\
\hline Deranged LFTs & 1 & 0 & 0 & 0 & 0 & 0 & 0 & 0 & 0 \\
\hline Other/unknown & 1 & 0 & 0 & 0 & 0 & 0 & 0 & 0 & 0 \\
\hline Neurological, total & 0 & 0 & 0 & 0 & 3 & 0 & 0 & 0 & I \\
\hline Headache & 0 & 0 & 0 & 0 & 1 & 0 & 0 & 0 & 0 \\
\hline Limb weakness & 0 & 0 & 0 & 0 & 1 & 0 & 0 & 0 & 0 \\
\hline Vasovagal syncope & 0 & 0 & 0 & 0 & 0 & 0 & 0 & 0 & $\mathrm{I}$ \\
\hline Other $^{d}$ & 0 & 0 & 0 & 0 & 1 & 0 & 0 & 0 & 0 \\
\hline Respiratory, total & 0 & 0 & 0 & 0 & 2 & 0 & 0 & 0 & 0 \\
\hline Shortness of breath & 0 & 0 & 0 & 0 & 1 & 0 & 0 & 0 & 0 \\
\hline Worsening of asthma & 0 & 0 & 0 & 0 & 1 & 0 & 0 & 0 & 0 \\
\hline Hematological, total & 0 & 0 & I & 0 & 0 & 0 & 0 & 0 & 0 \\
\hline Leukopenia & 0 & 0 & 1 & 0 & 0 & 0 & 0 & 0 & 0 \\
\hline General, total & 2 & 1 & 0 & 2 & 2 & 0 & 1 & 0 & I \\
\hline Fatigue & 0 & I & 0 & 1 & 0 & 0 & 0 & 0 & 0 \\
\hline Cramps & 0 & 0 & 0 & 1 & 0 & 0 & 0 & 0 & 0 \\
\hline Cold intolerance & 1 & 0 & 0 & 0 & 0 & 0 & 0 & 0 & 0 \\
\hline Insomnia & 1 & 0 & 0 & 0 & 0 & 0 & 1 & 0 & I \\
\hline Hypertension & 0 & 0 & 0 & 0 & 2 & 0 & 0 & 0 & 0 \\
\hline Other & 0 & 0 & 0 & 0 & 1 & 0 & 0 & 0 & 1 \\
\hline Epistaxis & 0 & 0 & 0 & 0 & 1 & 0 & 0 & 0 & 1 \\
\hline New skin lesion & 0 & 0 & 0 & 0 & 0 & 0 & 0 & 0 & 0 \\
\hline Number of side effects & 7 & 2 & I & 3 & 9 & 0 & 10 & 2 & 3 \\
\hline Number of hospitalizations & 0 & 0 & 0 & 0 & 0 & 0 & 1 & 0 & 0 \\
\hline Number of discontinuations & I & 0 & 0 & 0 & 0 & 0 & 0 & 0 & 0 \\
\hline \multicolumn{4}{|l|}{ Patients with side effects, \% } & \multicolumn{6}{|c|}{$13 / 20(65 \%)$} \\
\hline \multicolumn{4}{|c|}{ Patients with significant side effects, \% } & \multicolumn{6}{|c|}{$2 / 20(10 \%)$} \\
\hline
\end{tabular}

Notes: ${ }^{a}$ Tonsillitis definition includes sore throat and throat infection. ${ }^{\mathrm{b}} \mathrm{Chest}$ infection includes bacterial pneumonia and viral pneumonia. ${ }^{\mathrm{C}} \mathrm{Change}$ in bowel habit includes development of tarry stool and diarrhea. ${ }^{\mathrm{d}}$ Other neurological side effects include speech difficulty and numbness.

data demonstrating that MMF is safe to use for longer periods of up to 18 months.

Our study is limited by its retrospective nature, small patient numbers, and the empirical adjustment in dosing regimen. Patients sometimes did not have a comprehensive
TED assessment recorded when they were monitored in the immunosuppression clinic instead of the MTDTED clinics (where more complete TED assessments were performed) once their inflammation was more quiescent and their immunosuppression established. Guidance regarding 
optimal treatment duration may be helped by ongoing radiological MRI assessment of radiological measurements of orbital inflammation, ${ }^{20}$ as well as a more consistent use of validated TED quality of life instruments, such as the Graves' ophthalmopathy quality of life questionnaire (GOQOL). ${ }^{21}$ Additionally, combinations of treatment modalities were used in some individuals due to the realworld setting of this study; thus, it is not possible to solely attribute all long-term therapeutic effects to MMF alone. We would recommend a long-term prospective study be performed on patients who do not receive oral prednisolone nor orbital radiotherapy, to fully establish the therapeutic effects of MMF.

In conclusion, MMF is an effective and safe second-line immunosuppressive drug for moderate-severe and sightthreatening TED, but we found that a longer treatment period of up to 72 weeks, and higher doses than that previously established by randomized controlled studies, were needed.

\section{Acknowledgments}

The authors would like to thank Bhavini Dixit for her invaluable support within the immunosuppression clinic; and are grateful to the Imperial Open Access Fund for assistance with the open access cost of this publication.

\section{Author Contributions}

All authors made a significant contribution to the work reported, whether that is in the conception, study design, execution, acquisition of data, analysis and interpretation, or in all these areas; took part in drafting, revising or critically reviewing the article; gave final approval of the version to be published; have agreed on the journal to which the article has been submitted; and agree to be accountable for all aspects of the work.

\section{Funding}

No funding was received for this study.

\section{Disclosure}

The authors report no conflicts of interest for this work.

\section{References}

1. Perros P, Cromble A, Kendall-Taylor P. Natural history of thyroid associated ophthalmopathy. Clin Endocrinol (Oxf). 1995;42(1):45-50. doi:10.1111/j.1365-2265.1995.tb02597.x

2. Genere N, Stan M. Current and emerging treatment strategies for Graves' orbitopathy. Drugs. 2019;79(2):109-124. doi:10.1007/ s40265-018-1045-9
3. Bartalena L, Baldeschi L, Dickinson A, et al. Consensus statement of the European Group on Graves' Orbitopathy (EUGOGO) on management of graves' orbitopathy. Thyroid. 2008;18(3):333-346. doi:10.1089/thy.2007.0315

4. Ponto K, Pitz S, Pfeiffer N, Hommel G, Weber M, Kahaly G. Quality of life and occupational disability in endocrine orbitopathy. Dtsch Arztebl Int. 2009. doi:10.3238/arztebl.2009.0283

5. Wiersinga WM. Graves' orbitopathy: management of difficult cases. Indian J Endocrinol Metab. 2012;16(Suppl 2):S150-2. doi:10.4103/ 2230-8210.104026

6. Mourits M, Prummel M, Wiersinga W, Koornneef L. Clinical activity score as a guide in the management of patients with Graves' ophthalmopathy. Clin Endocrinol (Oxf). 1997;47(1):9-14. doi:10.1046/ j.1365-2265.1997.2331047.x

7. Bahn R, Gorman C. Choice of therapy and criteria for assessing treatment outcome in thyroid-associated ophthalmopathy. Endocrinol Metab Clin North Am. 1987;16(2):391-407. doi:10.1016/s0889-8529(18)30485-7

8. Holladay JT. Proper method for calculating average visual acuity. $J$ Refract Surg. 1997;13(4):388-391. doi:10.3928/1081-597X19970701-16

9. Lee V, Avari P, Williams B, Perros P, Dayan C. A survey of current practices by the British Oculoplastic Surgery Society (BOPSS) and recommendations for delivering a sustainable multidisciplinary approach to thyroid eye disease in the United Kingdom. Eye. 2019;34(9):1662-1671. doi:10.1038/s41433-019-0664-z

10. Smith T, Kahaly G, Ezra D, et al. Teprotumumab for thyroid-associated ophthalmopathy. $N$ Engl J Med. 2017;376 (18):1748-1761. doi:10.1056/nejmoa1614949

11. Douglas R, Kahaly G, Patel A, et al. Teprotumumab for the treatment of active thyroid eye disease. $N$ Engl J Med. 2020;382(4):341-352. doi:10.1056/nejmoa1910434

12. Ye X, Bo X, Hu X, et al. Efficacy and safety of mycophenolate mofetil in patients with active moderate-to-severe Graves' orbitopathy. Clin Endocrinol (Oxf). 2016;86(2):247-255. doi:10.1111/ cen. 13170

13. Kahaly G, Riedl M, König J, et al. Mycophenolate plus methylprednisolone versus methylprednisolone alone in active, moderate-to-severe Graves' orbitopathy (MINGO): a randomised, observer-masked, multicentre trial. Lancet Diabetes Endocrinol. 2018;6(4):287-298. doi:10.1016/s2213-8587(18)30020-2

14. Kelada M, Avari P, Farag S, et al. Association of other autoimmune diseases in thyroid eye disease. Front Endocrinol. 2021;12:116. doi:10.3389/fendo.2021.644200

15. Staatz C, Tett S. Clinical pharmacokinetics and pharmacodynamics of mycophenolate in solid organ transplant recipients. Clin Pharmacokinet. 2007;46(1):13-58. doi:10.2165/00003088200746010-00002

16. Behrend M. Adverse gastrointestinal effects of mycophenolate mofetil. Drug Saf. 2001;24(9):645-663. doi:10.2165/00002018200124090-00002

17. Riedl M, Kuhn A, Krämer I, Kolbe E, Kahaly G. Prospective, systematically recorded mycophenolate safety data in Graves' orbitopathy. J Endocrinol Invest. 2016;39(6):687-694. doi:10.1007/ s40618-016-0441-9

18. Aktaran Ş, Akarsu E, Erbağci İ, Araz M, Okumuş S, Kartal M. Comparison of intravenous methylprednisolone therapy vs. oral methylprednisolone therapy in patients with Graves' ophthalmopathy. Int $J$ Clin Pract. 2006;61(1):45-51. doi:10.1111/j.17421241.2006.01004.x

19. Marcocci C. Comparison of the effectiveness and tolerability of intravenous or oral glucocorticoids associated with orbital radiotherapy in the management of severe Graves' ophthalmopathy: Results of a Prospective, Single-Blind, Randomized Study. J Clin Endocrinol Metab. 2001;86(8):3562-3567. doi:10.1210/jc.86.8.3562 
20. Feeney C, Lingam R, Lee V, Rahman F, Nagendran S. Non-EPI-DWI for detection, disease monitoring, and clinical decision-making in thyroid eye disease. Am J Neuroradiol. 2020;41(8):1466-1472. doi:10.3174/ajnr.a6664
21. Terwee C, Gerding M, Dekker F, Prummel M, Wiersinga W. Development of a disease specific quality of life questionnaire for patients with Graves' ophthalmopathy: the GO-QOL. $\mathrm{Br}$ J Ophthalmol. 1998;82(7):773-779. doi:10.1136/bjo.82.7.773

\section{Publish your work in this journal}

Clinical Ophthalmology is an international, peer-reviewed journal covering all subspecialties within ophthalmology. Key topics include: Optometry; Visual science; Pharmacology and drug therapy in eye diseases; Basic Sciences; Primary and Secondary eye care; Patient Safety and Quality of Care Improvements. This journal is indexed on PubMed

Submit your manuscript here: https://www.dovepress.com/clinical-ophthalmology-journal
Central and CAS, and is the official journal of The Society of Clinical Ophthalmology (SCO). The manuscript management system is completely online and includes a very quick and fair peer-review system, which is all easy to use. Visit http://www.dovepress.com/ testimonials.php to read real quotes from published authors. 